ESJ Social Sciences

\title{
Rôle de la stabilité politique dans la promotion de l'investissement direct étranger en Afrique du Nord
}

\author{
El Khider Abdelkader \\ Zerouali Boukhal Imane
}

FSJES, Université Cadi Ayyad, Marrakech, Maroc

Doi:10.19044/esj.2021.v17n38p181

Submitted: 30 October 2020

Accepted: 29 October 2021

Published: 30 November 2021
Copyright 2021 Author(s)

Under Creative Commons BY-NC-ND

4.0 OPEN ACCESS

Cite As:

Abdelkader E-K. \& Imane Z.B. (2021). Rôle de la stabilité politique dans la promotion de l'investissement direct étranger en Afrique du Nord. European Scientific Journal, ESJ, 17 (38), 181. https://doi.org/10.19044/esj.2021.v17n38p181

\section{Résumé}

La stabilité politique a connu récemment un regain d'intérêt pour la manière dont elle affecte les décisions des investisseurs étrangers dans de nombreux pays en Afrique du Nord . L'objectif de cet article est de mettre en évidence le rôle crucial qu'elle joue dans l'attractivité des investissements directs étrangers, et d'analyser l'environnement politique des pays de cette région. Ainsi, à travers une évaluation empirique des données de panel, et selon le test d'Hausman, le modèle à effets fixes demeure le plus adapté pour notre analyse. Il a, ainsi, été conclu que la stabilité politique a un lien significatif et positif avec l'attractivité des investissements directs étrangers dans les cinq pays étudiés.

Mots clés : Stabilité politique, Investissement direct étranger, Afrique 


\title{
The Role of Political Stability in the Promotion of Foreign Direct Investment in North Africa
}

\author{
El Khider Abdelkader \\ Zerouali Boukhal Imane
}

FSJES, Université Cadi Ayyad, Marrakech, Maroc

\begin{abstract}
Recently, political stability has seen a resurgence of interest in how it affects the decisions of foreign investors in many countries in North Africa. The objective of this article is to highlight the crucial role it plays in the attractiveness of foreign direct investment, and to analyze the political environment of the countries of this region. Thus, through an empirical evaluation of the panel data, and according to the Hausman test, the fixedeffects model remains the most suitable for our analysis. It was thus concluded that political stability has a significant and positive link with the attractiveness of foreign direct investment in the five countries studied.
\end{abstract}

Keywords: Political stability, Foreign direct investment, Africa

\section{Introduction}

Dans les années 1950, l'investissement direct étranger (IDE) était considéré comme une menace et un facteur de dominance pouvant porter atteinte à l'économie du pays hôte (Alaya, 2004). Aujourd'hui, la donne a changé. Une large majorité des économistes présentent l'IDE comme étant le remède miracle pour les problèmes de croissance des pays en développement (Sachwald, 1995). Les efforts déployés par plusieurs pays pour attirer autant que possible d'IDE et la concurrence que se sont livrés ces mêmes pays pour accaparer d'importants flux des investissements internationaux, sont les preuves d'une conscience unanime de l'importance des IDE.

Par ailleurs, si le dynamisme du marché intérieur constitue l'un des conditions de base dans le choix du pays d'implantation, il existe d'autres facteurs qui commandent la décision finale de l'investisseur étranger. Il s'agit des fondamentaux macroéconomiques (croissance économique, ouverture commerciale, inflation, etc), de l'environnement politique (contrôle de la corruption, stabilité politique, niveau de démocratie), et bien d'autres déterminants.

Constituant l'assise indispensable au fonctionnement des rouages économiques, la stabilité politique fait partie des facteurs primordiaux de l'attractivité du pays et renforce sa compétitivité. Aharoni (2006) indique qu'une nation doit présenter un certain niveau de stabilité politique et 
économique avant même d'être considérée comme un site d'investissement. D'autres chercheurs, comme Vernon (1974), indiquent qu'il s'agit d'un facteur parmi tant d'autres ayant des effets relativement égaux sur les décisions d'investissement. Une troisième série d'études comme celle de Bauer (1967) indique que l'instabilité politique peut ne pas jouer un rôle aussi vital dans la décision d'investissement que le premier ensemble de références pourrait le suggérer.

Ainsi, vu qu'il existe plusieurs avis sur le sujet nous nous intéressons dans cet article à connaître le rapport de la stabilité politique avec l'attractivité des investissements directs étrangers à travers une étude empirique sur un échantillon de pays africains.

Dans le cadre de cette analyse, l'intérêt se porte sur le cas des pays d'Afrique du Nord car la majorité de ces pays se sont lancés dans un processus de réformes institutionnelles et politiques afin de créer un nouvel élan au développement et jeter les bases d'un nouveau mode de gouvernance. Ainsi, nonobstant les critiques parfois criardes quant à la qualité de leur environnements politiques (exemple du printemps arabe), ces pays continuent à recevoir du capital sous forme d'investissement direct étranger, c'est ainsi que la contribution dans les pages qui suivent vise à fournir un éclairage sur les concepts entourant la logique de la politique africaine.

Ce papier est organisé de la manière suivante : la première partie présente les principaux travaux évaluant le rapport entre la stabilité politique et l'attractivité de l'investissement direct étranger. Dans la deuxième le point est mis sur l'analyse de l'environnement politique en Afrique du Nord en donnant un aperçu sur les flux entrants des capitaux étrangers vers ce continent, et la troisième partie présente la méthodologie et résultats de notre étude empirique.

\section{Revue de littérature :}

Selon la Banque mondiale (1996), la stabilité politique est liée à la perspective de la stabilité du gouvernement ainsi qu'à l'absence de violence et de terrorisme à motivation politique. Il s'agit d'une situation dans laquelle les membres de la société se limitent largement à des comportements considérés comme étant dans les limites du rôle politique habituel. L'absence d'une telle atmosphère politique normale, qui menace la stabilité du gouvernement, est considérée comme une instabilité politique. Les conflits entre acteurs politiques et le terrorisme pourraient avoir recours à la violence et à l'instabilité du système politique (Enders et al, 2006). Ces derniers temps, les activités terroristes sont devenues répandues avec les effets qui en résultent sur les investissements et les activités économiques.

Ainsi, s'il existe plusieurs études sur les déterminants des IDE, moins d'attention a été accordée à l'impact des variables clés de l'environnement 
institutionnel telles que la stabilité politique sur l'IDE. Par conséquent, l'objectif de ce document dans ce domaine est une tentative de combler cet écart.

Pendant des années, le service conjoint du Foreign Investment Advisory Service, de la Société financière internationale et de la Banque mondiale s'est efforcé d'aider les gouvernements africains à promouvoir un environnement propice à l'investissement direct étranger. Le gouvernement latino-américain a été réceptif à ces conseils et il a fait d'énormes progrès dans l'amélioration du cadre de base de l'IDE dans les pays asiatiques (Blemmion, 2010). Cela a créé une tendance à la hausse en termes d'investissements directs étrangers dans le pays au cours des dernières années. Cependant, la baisse des IDE observée dans les pays africains entre 2008 et 2010 était due aux violences postélectorales observées après les élections générales de 2007. Ainsi, en 2012, une autre baisse des IDE a été enregistrée suite à l'incertitude des élections générales de 2013 où les investisseurs étrangers craignaient d'investir dans le pays en raison des pertes subies après les élections de 2007 (Amin, 2014). Cela indique que la stabilité politique est un facteur déterminant majeur de l'IDE dans un pays.

Dès lors, diverses recherches ont été menées sur l'investissement direct étranger et l'environnement politique. Gani et al. (2013) stipulent que les flux d'IDE nécessitent un environnement propice aux investissements pour prospérer dans le pays d'accueil. Le manque de stabilité politique a tendance à créer de l'appréhension et de l'incertitude, ce qui augmente le risque d'investissement apparent. Un tel risque sans une croissance potentielle correspondante du rendement attendu de l'investissement est susceptible de décourager les investissements étrangers dans le pays. L'instabilité politique nuit également à la croissance économique en nécessitant le détournement des ressources des activités économiques qui propulseraient la croissance vers d'autres domaines liés aux activités de renforcement de la sécurité. Ainsi, théoriquement, l'instabilité politique sape les entrées d'IDE ainsi que la croissance économique.

Lucas (1990) soutient que seul le risque politique est un facteur important pour limiter l'entrée des flux de capitaux au sein d'un pays. Il est à signaler, ainsi, que l'examen empirique de la relation entre la stabilité politique et l'IDE est lié aux travaux pionniers de Basi (1966) qui a établi le rôle de la stabilité politique dans l'attractivité des entrées d'IDE. Des études ultérieures ont généré des résultats contradictoires dans différents contextes en utilisant diverses approches. Malgré le nombre considérable d'études administrées sur cette relation, il existe un désaccord évident parmi les chercheurs sur l'existence ainsi que la direction du lien entre la stabilité politique et les IDE.

Adeoye (2009), Wernick et al. (2009) ainsi que Kim (2010) ont soutenu que la stabilité politique encourage les entrées d'IDE dans de 
nombreux pays. Un autre groupe, par exemple Lucke et Eichler (2016), Yang et al. (2018), Luiz et Ruplal (2013), ont suggéré que la stabilité politique décourage les entrées d'IDE dans divers pays. Dans une autre soumission, le troisième groupe de chercheurs, par exemple, Gani et Al-Abri (2013), Okafor (2015), Akhtar (2000) et Parinduri et al. (2011) ont affirmé qu'il n'y a pas de relation significative entre la stabilité politique et les IDE dans plusieurs contextes.

L'absence d'un consensus concernant la relation pourrait être associée à diverses mesures de stabilité politique utilisées par différentes études pour divers pays. De leur côté, Salako et Adebusuyi (2001) ont utilisé le processus d'estimation OLS pour explorer le rôle des variables macroéconomiques et de l'environnement politique sur les entrées d'IDE au Nigeria. Dans le même ordre d'idées, l'étude des déterminants des IDE au Pakistan par Akhtar (2000) à partir d'une analyse OLS, couvrant la période 1972-1996, confirme un lien non significatif entre la stabilité politique, la croissance du marché et l'IDE.

D'un autre côté, Rashid et al. (2017) ont remis en question l'effet de la stabilité politique ainsi que certaines variables macroéconomiques clés sur l'IDE en utilisant le modèle à effet fixe (FEM) avec un ensemble de données de 14 ans des 15 principaux pays d'Asie-Pacifique considérés comme les économies les plus compétitives de la région. Leur résultat implique que les pays de l'Asie-Pacifique doivent promouvoir la stabilité de leur environnement institutionnel et macroéconomique, car la stabilité politique, l'ouverture commerciale et le PIB ont une influence positive et significative sur les entrées d'IDE.

Dans une autre étude, Morrissey et Udomkerdmongkol (2012) ont appliqué la technique GMM pour explorer le lien entre les variables institutionnelles, l'investissement privé et l'IDE dans 46 économies en développement échantillonnées au cours de la période 1995-2009. Ces chercheurs ont documenté à partir de leur analyse, que la stabilité politique exerce une influence positive sur l'afflux d'IDE de manière significative.

Diverses recherches varient en fonction du contexte et de la période de l'étude ainsi que la méthodologie employée dans leurs enquêtes. Tout cela pourrait aboutir à différents résultats et conclusions. Ainsi, il n'y a pas de consensus clair sur l'impact positif de la stabilité politique sur l'IDE, et plus encore, peu de travaux ont essayer d'examiner ce lien dans les pays de l'Afrique.

\section{IDE et stabilité politique en Afrique du Nord}

Les bouleversements politiques et sociaux qui ont suivi le printemps arabe de 2011 continuent de dominer l'activité économique et les perspectives à court terme en Afrique du Nord selon la Banque Mondiale. Bien que les transitions politiques promettent une plus grande liberté politique et 
économique, dans la région, le processus est loin d'être achevé et s'est accompagné d'une instabilité politique et macroéconomique accrue en 2013. En Égypte par exemple, la montée des tensions sociales et politiques a fortement pesé sur la confiance des investisseurs.

Une image plus désagrégée de l'IDE dans la région de l'Afrique du Nord montre quelques différences par rapport au reste du monde et au fil du temps. Bien que la région ait attiré plus d'IDE dans les années 2000 par rapport aux années 1990, reflétant l'amélioration de l'environnement des affaires dans de nombreuses économies, la majorité des pays ont enregistré des performances inférieures à leur potentiel.

En outre, les pays en développement importateurs de pétrole n'ont reçu que 30 pour cent des entrées d'IDE de la région et une grande partie provenait des économies du CCG. Lorsque les prix du pétrole ont augmenté dans les années 2000, les pays sources ont réorienté leurs investissements vers les exportateurs de pétrole de la région. Après 2010, les entrées d'IDE ont diminué dans toute la région, l'investissement public a diminué dans les pays en développement de l'Afrique du Nord et le Moyen Orient, tandis que l'investissement privé intérieur est resté relativement inchangé.

La question de savoir si la baisse des IDE après 2011 est due à l'instabilité politique n'est pas claire, tout comme le montre la littérature. En outre, les flux d'IDE vers les secteurs à forte intensité de ressources et non échangeables semblent immunisés contre l'instabilité politique, mais les flux d'IDE dans les secteurs échangeables affichent une réponse clairement négative. Enfin, les conditions économiques ont continué à jouer un rôle important pour attirer les IDE (Banque Mondiale, 2013).

\section{II.1. Analyse de l'environnement politique en Afrique du Nord :}

Dans son rapport de 2019, la Banque africaine de développement, avance que la stabilité politique devrait avoir un impact sur le développement économique, notamment en Libye, et dans d'autres pays dotés d'un secteur du tourisme important, comme l'Égypte, le Maroc et la Tunisie. Elle influence également les perceptions du risque et donc les investissements (African Development Bank Group ${ }^{2019)}$. Ainsi, les enquêtes qui sous-tendent l'indice de compétitivité mondial indiquent que la corruption, la lourdeur de la bureaucratie, et l'instabilité politique sont les principales lacunes de l'Afrique du Nord (Forum économique mondial, 2018). L'instabilité politique est cyclique et prise en compte en raison des troubles qui secouent la région depuis 2011. Cependant, plusieurs tentatives de réforme de l'environnement politique ont été entamées par les preneurs de décisions dans les différents pays de la région :

Libye: Une instabilité politique et sécuritaire, sévit toujours en Libye depuis le printemps Arabe. L'indice des États fragiles du Fonds pour la Paix 
de 2018 a classé la Libye au 25ème rang parmi les 178 pays les plus fragiles (OCDE, 2018). Le pays a vu la mise en place de deux gouvernements rivaux en août 2014, entraînant une forte escalade de la violence et une paralysie effective en termes de services fournis par l'État. Les groupes extrémistes ont pu profiter de l'incertitude dans le pays pour renforcer leur position et constituer une menace croissante pour l'État. Au même temps, le réseau complexe de relations de la Libye au niveau local a conduit à des conflits locaux qui maintiennent leur propre dynamique tout en influençant l'environnement politique au sens large. Après la révolution, les intérêts politiques dans le pays sont restés fragmentés, avec peu de consensus sur ce à quoi devrait ressembler la Libye post-Kadhafi. Le premier courant a prôné une rupture forte avec le passé et l'héritage de l'ancien régime, et a cherché à protéger les «principes de la révolution». Le deuxième courant, sans être contre-révolutionnaire ou pro-Kadhafi, promeut une vision pour la Libye qui n'exclut pas les parties du pays qui ont soutenu l'ancien régime et qui peuvent s'appuyer sur les capacités existantes de l'État libyen.

Les deux gouvernements libyens, le gouvernement de la chambre des représentants $(\mathrm{HoR})$ à Bayda et le gouvernement du congrès national général (GNC) à Tripoli, sont tous deux soutenus par divers groupes armés et communautés locales. Au cœur de la querelle se trouvent des idées contestées pour l'avenir du pays difficilement conciliables, encore compliquées par les intérêts personnels, la force des loyautés issues des réseaux sociaux, les griefs intercommunautaires et la concurrence pour l'accès aux ressources économiques (UNDP, 2015).

(Mohamed Eljarh, 2018), stipule que le conflit en Libye est alimenté par un réseau d'intérêts internes et externes entrelacés. Les groupes armés et politiques concurrents de la Libye sont peu incités à s'engager dans de véritables efforts de réconciliation politique car ils bénéficient d'un soutien substantiel de l'étranger. Au niveau local, la fracture institutionnelle qui implique des institutions libyennes clés telles que la Banque centrale et la National Oil Corporation a été particulièrement dommageable car elle a privé le pays de ressources économiques et financières essentielles. Surmonter les obstacles internes au renforcement des institutions et, par extension, à la paix et à la stabilité en Libye nécessite une approche holistique qui intègre les acteurs internes aux niveaux local et national.

Egypte : Les dix dernières années ont connu des formes de perturbations politiques en Égypte qui étaient pratiquement inimaginablesdepuis le mouvement des manifestations de 2011 ; l'élection en 2012 du candidat des Frères musulmans Mohammed Morsi à la présidence égyptienne ; le coup d'État de 2013 qui a renversé Morsi ; et l'accession officielle au pouvoir en 2014 de l'actuel président égyptien Abdel Fattah al-Sisi -. Peu d'analystes seraient en désaccord avec l'affirmation selon laquelle les 
circonstances démographiques et les développements technologiques ont joué un rôle crucial dans le déclenchement et le maintien du mouvement populaire qui a déclenché cette chaîne d'événements.

Pourtant, malgré tous les changements politiques intervenus, les dix dernières années ont également été marquées par d'importantes formes de continuité. D'un point de vue institutionnel, l'Égypte reste une autocratie avec un régime politique soutenu par une armée puissante et économiquement influente. Et d'un point de vue démographique, bon nombre des mêmes défis auxquels l'Égypte a été confrontée il y a une décennie (comme le taux de chômage relativement élevé), continuent d'être des préoccupations pertinentes aujourd'hui.

Dans les années qui ont suivi le renversement de Morsi, l'Égypte a connu des tendances de plus en plus autocratiques sous la direction du président al-Sisi. Ce dernier a donné carte blanche au ministère égyptien de l'Intérieur pour perpétrer des abus généralisés, notamment des arrestations arbitraires et des actes de torture contre des dissidents présumés, dans le but de créer une stabilité politique. Les membres et sympathisants présumés des Frères musulmans ont été particulièrement ciblés, entrainant l'emprisonnement de militants des Frères musulmans et la réduction significative des activités de service social du groupe.

Les Égyptiens jouissent aujourd'hui de moins de libertés personnelles qu'à l'époque de Moubarak. Des milliers de blogueurs, journalistes et militants politiques égyptiens ont été emprisonnés ; les sites Web ont été interdits et les médias sont sous contrôle strict. Une loi signée en août 2018 a renforcé le contrôle du gouvernement sur Internet, plaçant des milliers de comptes de médias sociaux sous surveillance gouvernementale (Feingold, 2018). Al-Sisi s'est appuyé sur des acteurs externes, y compris les États du Golfe, pour soutenir ses politiques de plus en plus autoritaires. Un bloc d'États contrerévolutionnaires à travers le monde arabe, dont l'Égypte, l'Arabie saoudite et les Émirats arabes unis, a doublé l'autoritarisme. En fait, des milliers d'Égyptiens se sont expatriés pour des raisons politiques, craignant les condamnations judiciaires, les poursuites, les attaques dans les médias et la menace d'agression physique. Et les Égyptiens qui sont partis représentent tous les horizons de la vie politique, y compris les gauchistes, les libéraux ainsi que les islamistes. La législature égyptienne n'a jamais été un contrepoids particulièrement efficace à l'exécutif ; c'est encore plus le cas aujourd'hui que par le passé. En février 2019, le parlement égyptien a approuvé un processus d'organisation d'un référendum susceptible de permettre à al-Sissi de prolonger son règne jusqu'en 2034, renforçant ainsi son régime personnel et son régime militaire en Égypte, de manière plus générale (Blaydes, ${ }^{2019)}$.

Tunisie : Dix ans après le printemps arabe, alors que ses voisins se sont effondrés dans la guerre civile ou la dictature renouvelée, la Tunisie a brisé le 
moule, passant à la démocratie en 2011 et la maintenant depuis. Aujourd'hui, le président tunisien Kais Saied est le seul chef d'État de la région à pouvoir prétendre avoir remporté des élections libres et équitables. Certains prétendent que le succès politique de la Tunisie est dû au fait qu'il est un petit pays homogène avec un niveau de développement élevé, une population bien éduquée et avec une culture de tolérance. Mais en 2013, malgré ces qualités, la transition tunisienne était au bord de l'effondrement, avec deux assassinats politiques, une forte polarisation politique et la suspension de la seule institution élue du pays. L'opposition tunisienne, inspirée par le coup d'État égyptien, est descendue dans la rue, appelant à la chute du gouvernement tunisien démocratiquement élu.

Cette crise a mis en évidence les véritables atouts de la Tunisie. L'armée et les forces de sécurité sont restées en dehors de la mêlée, les partis politiques se sont réunis pour trouver un consensus et des institutions civiques bien développées ont aidé à arbitrer le dialogue. La Tunisie a depuis approuvé une constitution progressiste et a organisé deux tours supplémentaires d'élections libres en 2014 et 2019. Beaucoup considèrent aujourd'hui la Tunisie comme le pays le plus démocratique du Moyen-Orient.

Mais ironiquement, les mêmes facteurs qui ont aidé la transition de la Tunisie à survivre à ses premières années ont depuis entravé sa transition. Chacun de ces facteurs ont, à leur manière, entravé la consolidation de la démocratie tunisienne. La petite armée et le manque de coordination avec les forces de sécurité ont créé un vide sécuritaire, permettant une attaque contre l'ambassade des États-Unis en 2012, deux assassinats politiques et des attaques majeures de l'État islamique en 2015. Ces attaques terroristes ont freiné la volonté politique de poursuivre la réforme du secteur de la sécurité, permettant aux forces de police tunisiennes de continuer à commettre des abus qui alimentent les griefs envers le système politique. Ces attaques ont également entraîné des coûts économiques importants pour le secteur du tourisme, tout en redirigeant le budget vers les ministères de l'intérieur et de la défense. Aux prises avec la faiblesse du secteur de la sécurité, les politiciens ont largement laissé de côté les demandes économiques de justice sociale qui ont alimenté la révolution de 2011.

La Tunisie est peut-être la seule démocratie à émerger du printemps arabe, mais des défis majeurs subsistent, notamment des brutalités policières et une désillusion à l'égard du système. Paradoxalement, chacun de ces défis peut avoir été exacerbé par les facteurs mêmes qui ont aidé la démocratie tunisienne à survivre dans les premières années de sa transition. Sa recherche de consensus, son secteur de la sécurité faible et sa société civile puissante contribuent à expliquer à la fois pourquoi la Tunisie a réussi et pourquoi elle ne s'est pas encore consolidée (Grewal, 2021).

Maroc: La situation politique du Maroc est plutôt stable, le roi 
Mohammed VI détenant l'essentiel du pouvoir politique entre ses mains, tandis que les pouvoirs du parlement et du gouvernement sont limités. Il n'y a pas de menace immédiate pour la monarchie, car le roi est populaire auprès du peuple.

En 2011, alors que le «printemps arabe » se répercutait dans une grande partie de la région, le Maroc a connu quelques protestations, mais celles-ci étaient à relativement petite échelle par rapport aux autres pays touchés. On a ainsi abouti à l'adoption par le roi d'une constitution plus progressiste et à l'élection d'un nouveau gouvernement la même année.

Les prochaines élections législatives sont prévues en septembre 2021. L'actuel gouvernement de coalition à 12 partis est très fragmenté. Le processus de normalisation du Maroc avec Israël reste controversé et a conduit à la démission de plusieurs membres du principal parti de la coalition, le PJD. Malgré un soutien accru à l'opposition, le PJD islamo-démocrate conservateur devrait rester le plus grand parti au parlement après les élections.

Cependant, le Maroc reste vulnérable à la menace de l'extrémisme islamique. Les combattants marocains de retour de Libye et de Syrie inquiètent particulièrement les autorités. Cependant, le pays n'a pas été touché par des attaques terroristes majeures au cours des dernières années. La préservation de la sécurité est une priorité du gouvernement, étant donné l'importance des revenus du tourisme pour l'économie. Un autre problème de sécurité est le conflit au Sahara occidental entre le Maroc et le Front Polisario, avec des combats qui ont récemment repris, mettant fin à un cessez-le-feu vieux de 29 ans.

Algérie: L'accession d'Abdelmadjid Tebboune à la présidence était censée apporter une certaine prévisibilité aux dirigeants militaires algériens. Mais depuis qu'il a été déclaré vainqueur de l'élection présidentielle en décembre 2019, le régime est entré dans une nouvelle phase d'incertitude. La pandémie de COVID-19, la dissidence continue, la volatilité politique et l'aggravation du malaise économique ont affecté les calculs de l'oligarchie au pouvoir. Deux ans après le début du soulèvement populaire en Algérie, connu sous le nom de mouvement Hirak, le pays est coincé dans la même impasse à laquelle il est confronté depuis 2019.

Après des années de stagnation politique, le mouvement Hirak, qui a éclaté en février 2019 contre la candidature du président de l'époque Abdelaziz Bouteflika pour un cinquième mandat, a changé la dynamique des relations entre l'oligarchie au pouvoir en Algérie et sa population. Bien qu'il n'ait pas entraîné de profonds changements dans les structures gouvernantes du pays, le soulèvement pacifique a abouti à la destitution de Bouteflika par l'armée en avril 2019. Et bien que les manifestants aient continué à se rassembler régulièrement dans les rues de la plupart des villes du pays pour contester l'armée retranchée, le régime a réagi par le biais de mécanismes institutionnels. 
Cependant, à ce jour, ces réponses se sont avérées insuffisantes pour endiguer le mécontentement populaire croissant (Serrano, 2021).

Ainsi, il est peu probable que le mouvement Hirak puisse imposer une trajectoire en Algérie qui imite l'expérience tunisienne de 2011. La perspective d'un changement de régime révolutionnaire déclenché par la révolte populaire telle que poursuivie par le mouvement Hirak naissant au printemps 2019 repose sur une fausse comparaison. Le pouvoir et la politique en Algérie diffèrent grandement de la Tunisie voisine. Au fil du temps, et dans le sillage de la pause de mobilisation liée à la pandémie à partir de mars 2020, le mouvement Hirak semble avoir pris conscience amèrement que le régime militaire algérien n'a rien à voir avec l'État policier de Ben Ali. L'Algérie ressemble plus au régime de Maduro au Venezuela de l'autre côté de l'Atlantique, soutenu par la rente militaire et pétrolière, qu'à la Tunisie voisine (EuroMed Droit, 2021). L'avenir politique de l'Algérie dépend dans une large mesure de l'armée. Le haut commandement de l'armée se considère comme l'épine dorsale de l'État algérien, déterminé à assurer la sécurité du pays et à protéger sa forme républicaine de gouvernement (Cook, 2007).

\section{II.2. Analyse des flux d'IDE dans les pays d'Afrique du Nord}

Dans la région de l'Afrique du Nord, au début des années 90, les gouvernements ont pris des mesures pour ouvrir l'économie et libéraliser le système financier afin d'établir un environnement propice à l'investissement. Cependant, malgré les quelques progrès en termes de stabilité macroéconomique et de réformes structurelles, ces pays connaissent encore des difficultés en termes d'attractivité des investissements étrangers.

Indépendamment des biens communs culturels et historiques entre les pays d'Afrique du Nord, il existe d'abondantes variations en de développement économique, social et politique. Alors que les ressources naturelles représentent plus de $95 \%$ des exportations de l'Algérie et de la Libye, elles ne représentent qu'environ $13 \%$ au Maroc et en Tunisie.

Selon la Banque mondiale (2011), le montant des flux d'IDE dans les pays d'Afrique du Nord est passé d'une moyenne annuelle de 2,2 milliards de dollars américains dans les années 90 à 12,5 milliards de dollars américains dans les années 2000.

Compte tenu de la taille de ses ressources naturelles et de sa situation géographique stratégique, on peut facilement affirmer que les flux d'IDE vers la région d'Afrique du Nord sont encore insuffisants par rapport à ceux d'autres régions en développement. La moyenne du stock d'IDE par rapport au PIB au cours de la période 1996-2017 dans la région de l'Afrique du Nord était de $25,7 \%$ contre $47,3 \%$ dans la région de l'Afrique australe et $49,7 \%$ dans la région de l'Asie du Sud-Est 
Figure 1 : Flux d'IDE dans les pays d'Afrique du Nord (milliards USD)

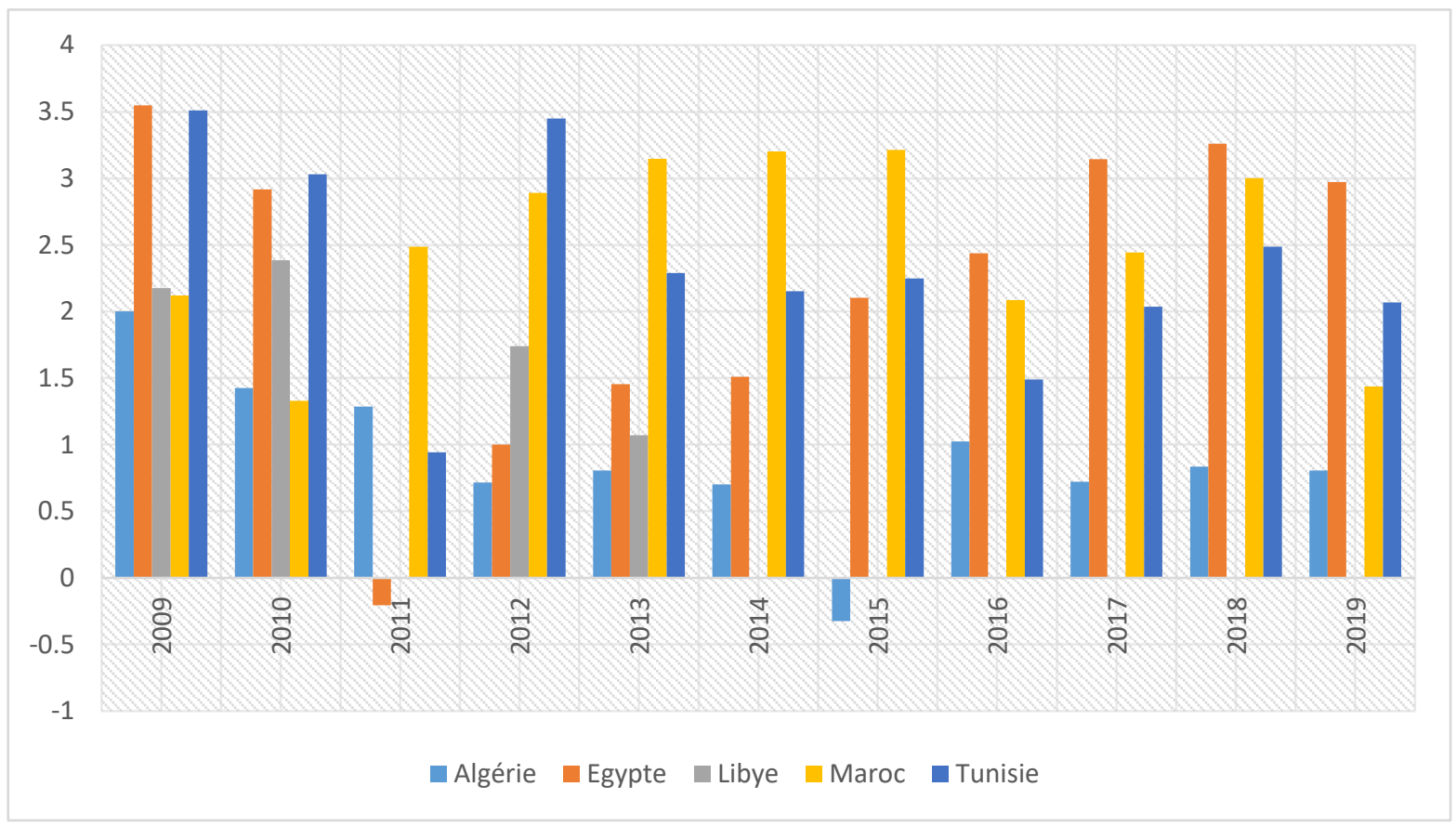

Source : Banque mondiale

L'Égypte a connu une volatilité considérable dans ses entrées d'IDE au cours de la dernière décennie. Les flux nominaux d'IDE ont culminé en 2007 à 11,6 milliards USD, soit l'équivalent de 8,4\% du PIB, avant de décliner à la suite de la crise financière mondiale. La révolution égyptienne de 2011 a entraîné une baisse encore plus brutale des entrées d'IDE. Les flux se sont ensuite progressivement redressés, atteignant 8,1 milliards USD en 2016. En ne considérant que les IDE entièrement nouveaux, les flux ont affiché une volatilité similaire, combinant des pics (2009 et 2018) et des creux (2011 et 2012), se succédant parfois rapidement. Les mouvements des flux entrants de l'Égypte ont été considérablement plus prononcés que ceux de la région de l'Afrique du Nord dans son ensemble.

Entre 2009 et 2010, les entrées d'IDE en Libye étaient à peu près conformes aux niveaux de la région. En 2007, les entrées ont culminé à 4,7 milliards USD, soit l'équivalent de $7 \%$ du PIB, et bien au-dessus de la moyenne de la région MENA et d'autres économies en développement. Aucune donnée sur les IDE n'est disponible pour la période suivant l'intervention dirigée par l'OTAN pour chasser Kadhafi en 2011 dans la base de données de la balance des paiements du FMI. Cependant, les données de la CNUCED montrent que les entrées d'IDE ont atteint un creux en 2014 et se sont à peine redressées depuis lors.

Pour la plupart des pays étudiés, les entrées d'IDE ont été très volatiles 
par rapport à d'autres régions. Selon la banque mondiale, une grande partie de cette volatilité est une conséquence de l'instabilité politique que connaissent ces pays depuis le printemps arabe en 2011.

\section{Méthodologie et résultats :}

L'objectif de cet article est de tester l'importance de la stabilité politique dans l'attractivité des investissements directs étrangers en Afrique du Nord.

La variable dépendante dans notre modèle empirique est les flux des IDE entrants. Les variables explicatives sont l'indice de la stabilité politique, la croissance du PIB qui nous sert de prédicteur de la taille de marché et l'inflation. L'ensemble des données utilisées dans cette analyse est un ensemble de panel, disponible à partir des données de la Banque Mondiale. Ainsi, la période étudiée est de dix années ; de 2009 à 2019.

Le modèle se présente comme suit :

Où :

$$
\mathrm{IDE}_{\mathrm{i}, \mathrm{t}}=\boldsymbol{\beta}_{\mathbf{0}}+\boldsymbol{\beta}_{\mathrm{j}} \mathrm{PStab}_{\mathrm{i}, \mathrm{t}}+\boldsymbol{\beta}_{2} \mathrm{PIB}_{\mathrm{i}, \mathrm{t}}+\boldsymbol{\beta}_{\mathrm{j}} \operatorname{Inf}_{\mathrm{i}, \mathrm{t}}+\boldsymbol{\delta}_{\mathrm{i}, \mathrm{t}}
$$

$\mathrm{i}$ : est l'indice du pays

$\mathrm{t}$ : est l'indice du temps

$\beta_{0}$ : est le terme d'effet non observé spécifique au pays.

Le tableau 1 descriptif de l'échantillon, montre que le panel est équilibré. Ainsi, les flux entrants d'IDE dans les pays africains étudiés est significativement faible. Sa moyenne pour la période sous revue est de $1.71 \%$ du PIB, avec un écart type de 1.13. L'IDE minimum en pourcentage du PIB est de $-0.32 \%$, tandis que le maximum était de $3.54 \%$.

Tableau 1 : Statistiques descriptives de l'échantillon

\begin{tabular}{|c|c|c|c|c|c|}
\hline Variable & Obs & Mean & Std. Dev. & Min & Max \\
\hline IDE & 55 & 1.719762 & 1.132853 & -.3240123 & 3.548351 \\
\hline PStab & 55 & 19.03917 & 14.47468 & 1.428571 & 76.30331 \\
\hline PIB & 55 & 3.505951 & 19.55837 & -62.07592 & 123.1396 \\
\hline Inf & 55 & 4.503532 & 9.477967 & -25.3128 & 22.93255 \\
\hline \hline
\end{tabular}

Le test de spécification d'Hausman est ainsi utilisé pour déterminer lequel du modèle à effets fixes et à effets aléatoires est le mieux adapté pour cette analyse. L'hypothèse nulle pour le test de spécification d'Hausman montre une préférence pour le modèle à effets aléatoires par rapport au modèle à effets fixes.

Le tableau 2 présente les résultats d'estimation du modèle. Tout d'abord, nous effectuons des estimations en utilisant le modèle à effets fixes (FE) et le modèle à effets aléatoires (RE). Les résultats du test d'Hausman valident le FE pour tout l'échantillon. Ainsi, les résultats indiquent qu'il existe 
une relation positive et significative entre les flux d'IDE entrants et la stabilité politique dans les pays cinq pays étudiées.

\begin{tabular}{l}
\multicolumn{4}{|l|}{ Tableau 2 : Régression à effets fixes } \\
$\qquad$\begin{tabular}{|l|l|l|}
\hline \multicolumn{1}{|l|}{ Méthode d'estimation : Régression à effets fixes } \\
\hline Variables & Coefficients & Ecart-type \\
\hline PStab & $0.036771 * * *$ & 0.0084533 \\
\hline PIB & 0.0100505 & 0.0051464 \\
\hline Inf & 0.0101725 & 0.0125985 \\
\hline Constante & $0.9386242 * * *$ & 0.2075682 \\
\hline Nombre d'observations & 55 & \\
\hline R$^{2}$ between & 0.5277 & \\
\hline Wald chi2 (3) & 31.50 & \\
\hline Prob> chi2 & 0.0000 & \\
\hline Sargan- Hansen statistic & 10.06 & \\
\hline p-value & 0.0736 & \\
\hline ***significativité à 1\% $\%$ significativité à 5\% & \\
\hline
\end{tabular}
\end{tabular}

\section{Conclusion}

Les résultats de cette étude montre une relation positive est significative entre les entrées d'IDE et la stabilité politique. Ils s'inscrivent ainsi dans la même lignée que Groznykh et al. (2020) qui stipulent que les économies du monde préfèrent investir dans les pays d'Afrique en raison d'un meilleur contrôle de la corruption, d'indicateurs d'inclusion plus élevés, de meilleurs profils d'investissement et de politiques extérieures efficaces.

Après une analyse appropriée des résultats de la recherche, on peut conclure que si les économies d'Afrique du Nord recherchent une augmentation des investissements directs étrangers, il est alors impératif qu'elles améliorent l'environnement politique et offrent des conditions favorables aux pays investisseurs. Cependant, les la taille du marché et l'inflation semblent être inutiles pour les flux d'IDE dans les pays de la région, par conséquent, cela signifie que d'autres facteurs pourraient être plus importants pour ce groupe de pays en plus de la stabilité politique.

De plus, seule une variable décrivant la stabilité politique a été utilisée dans cette recherche. Cette variable est jugée capable de mieux décrire l'environnement politique des pays, même si elle a ses limites. Les auteurs prévoient de traiter ce problème en élargissant la base de données et en ajoutant des indicateurs quantitatifs et des variables factices, ce qui peut affiner les résultats de la recherche empirique.

\section{References:}

1. Adeoye, A. (2009). Macro-economic level corporate governance and FDI in emerging markets: Is there a close relationship? Journal of Economics and International Finance, 1(2), 30-43 
2. African Development Bank Group, (2019), Perspectives économiques en Afrique du Nord 2019.

3. Aharoni, Y. (1966). The foreign investment decision process. Boston: Division of Research, Graduate School of Business Administration, Harvard University.

4. Akhtar, M. H. (2000). The determinants of foreign direct investment in Pakistan: An econometric analysis. The Lahore Journal of Economics, 5(1), 1-22.

5. Alaya M, (2004), Investissement direct étranger et croissance économique: Le cas de la Tunisie, C.E.D, Université MontesquieuBordeaux IV

6. Amin, M (2014), Foreign Direct Investment and Economic Growth Literature Review from 1994 to 2012, Procedia Social and Behavioral Sciences, Volume 129, 15 May 2014, Pages 206-213, Available at: http://www.sciencedirect.com/ science/article/pii/S187704281402850X

7. Basi, R. (1966). Determinants of US direct investment in foreign countries. Kent, $\mathrm{OH}$ : Kent University Press.

8. Bauer, Raymond A., (1967), Ithiel de Sola Poole, and Lewis Anthony Dexter. American Business and Public Policy. New York: Atherton

9. Blaydes, L, (22 Avril 2019), Challenges to Stability in Egypt, Hoover Institution, Spring Series, Issue 519.

10. Blemmion, S. (2010). A review of the empirical literature on FDI determinants. Atlantic economic review. Austria press. Australia

11. Eljarh, M, (2018), The Libyan Crisis: Internal Barriers to Conflict Resolution and the Role of Multilateral ${ }^{1}$ Eljarh, M, (2018), The Libyan Crisis: Internal Barriers to Conflict Resolution and the Role of Multilateral Cooperation

12. Enders, W., Sachsida, A., \& Sandler, T. (2006). The impact of transnational terrorism on US foreign direct investment. Political Research Quarterly, 59(4), 517-531

13. EuroMed Droit, (2021), Proceedings from the HRDC consortium webinars Compiled and edited by Luis Martinez \& Rasmus Alienus Boserup.

14. Forum économique mondial. (2018). The Global Competitiveness Report 2017-2018

15. Gani, A., \& Al-Abri, A. S. (2013). Indicators of business environment, institutional quality and foreign direct investment in Gulf Cooperation Council (GCC) countries. International Review of Applied Economics, 27(4), 515-530.

16. Gani, A., \& Al-Abri, A. S. (2013). Indicators of business environment, institutional quality and foreign direct investment in Gulf Cooperation 
Council (GCC) countries. International Review of Applied Economics, 27(4), 515-530.

17. Grewal, S, (22 Janvier 2021), Ten years in, Tunisian democracy remains a work in progress, Order from Chaos

18. Groznykh, R, Mariev, O, Plotnikov, S , Fominykh, M, (2020), The role of political stability in foreign direct investment attraction: crosscountry analysis, Cbu International Conference On Innovations In Science And Education 2020 (Economics And Business), Prague, Czech Republic

19. Hur, J., Parinduri, R. A., \& Riyanto, Y. E. (2011). Cross-Border M\&A inflows and quality of country governance: Developing versus developed countries. Pacific Economic Review, 16(5), 638-655.

20. Kim, H. (2010). Political stability and foreign direct investment. International Journal of Economics and Finance, 2(3), 59-71

21. Lucas, Robert E., Jr. (1990). Why does not Capital Flow from Rich to Poor Countries. American Economic Review 80: 92-6

22. Lucke, N., \& Eichler, S. (2016). Foreign direct investment: The role of institutional and cultural determinants. Applied Economics, 48(11), 935-956.

23. Luiz, J. M., \& Ruplal, M. (2013). Foreign direct investment, institutional voids, and the internationalization of mining companies into Africa. Emerging Markets Finance and Trade, 49(4), 113-129.

24. Morrissey, O., \& Udomkerdmongkol, M. (2012). Governance, private investment and foreign direct investment in developing countries. World Development, 40(3), 437-445.

25. OCDE (2018), États de fragilité 2018, Éditions OCDE, Paris. https://doi.org/10.1787/9789264308916-fr

26. Okafor, G. (2015). Locational determinants of us outward FDI into Sub-Saharan Africa. The Journal of Developing Areas, 49(1), 187-205.

27. Rashid, M., Looi, X. H., \& Wong, S. J. (2017). Political stability and FDI in the most competitive Asia Pacific countries. Journal of Financial Economic Policy, 9(02), 140- 155.

28. Sachwald, F, (1995), Politique étrangère, 60(3), 776-778. Retrieved September 5, 2021, from http://www.jstor.org/stable/42713464

29. Salako, H. A., \& Adebusuyi, B. S. (2001). Determinants of foreign direct investment (FDI) in Nigeria: An empirical investigation. CBN Economic and Financial Review, 39(1), 20- 39.

30. See Cook, S. A. (2007). Ruling but not governing: The military and political development in Egypt, Algeria, and Turkey. The John Hopkins University Press.

31. Serrano, F, (17 Fevrier 2021), Two years after the start of Algeria's popular uprising, the regime is far from stable 
32. Spencer Feingold, (29 Août 2018), Egypt's President Sisi Ratifies New Internet Control Law, $C N N$.

33. UNDP, (2015), Instability and Insecurity in Libya.

34. Vernon, R., (1974), The Location of Economic Activity, in Economic Analysis and the Multinational Enterprise, ed. by John H. Dunning (London: Allen and Unwin)

35. Wernick, D. A., Haar, J., \& Singh, S. (2009). Do governing institutions affect foreign direct investment inflows? New evidence from emerging economies. International Journal of Economics and Business Research, 1(3), 317-332.

36. World Bank (1996). Governance. World Bank, Washington, DC. Retrieved from www.worldbank.org/governance/wgi/pdf/pv.pdf.

37. World Bank, (2011), Middle East and North Africa: Investing for Growth and Jobs, Economic Developments and Prospects Report, Office of the Chief Economist, Middle East and North Africa, the World Bank.

38. World Bank, (2013), Jordan Competitiveness and Innovation Partnership: Promoting Reforms at the National and Sectoral Levels, Finance and Private Sector Development, Middle East and North Africa, the World Bank

39. Yang, J. H., Wang, W., Wang, K. L., \& Yeh, C. Y. (2018). Capital intensity, natural resources, and institutional risk preferences in Chinese outward foreign direct investment. International Review of Economics \& Finance, 55, 259-272. 